\title{
AVALIAÇÃO DA QUALIDADE DE VIDA EM MULHERES COM FATORES DE RISCO CARDIOVASCULARES DE UMA UNIDADE BÁSICA DE SAÚDE
}

Aline Ferreira Lima Gonçalves ${ }^{1}$, Giovana Zago Cabrera ${ }^{1}$, Marilyn Garcia Silva ${ }^{1}$, Jéssica Almeida Andrade $^{1}$, Renata Augusta Beloni Digiovani ${ }^{2}$, Ilse de Lima Aruda Storel ${ }^{3}$, Sueli Aparecida Montonholi Bueno ${ }^{3}$, Eliani Zamberlan Zambrini ${ }^{4}$, Francis Lopes Pacagnelli ${ }^{2}$

${ }^{1}$ Discente, ${ }^{2}$ Docente do Curso de Fisioterapia, ${ }^{3}$ Docente do Curso de Medicina da UNOESTE, Presidente Prudente, SP;

${ }^{4}$ Educadora Física da Secretaria de Assistência Social de Presidente Prudente, SP.

Correspondência para: Francis Lopes Pacagnelli - francispacagnelli@unoeste.br

\section{RESUMO}

A maior prevalência de cardiopatias está em indivíduos do sexo masculino, porém, dados epidemiológicos mostram que as mulheres estão alcançando índices cada vez maiores. $\mathrm{O}$ objetivo deste trabalho foi orientar e atuar por meio de um programa de reabilitação cardiovascular em mulheres com fator de risco cardiovascular inseridas em uma Unidade Básica de Saúde (UBS) e avaliar sua qualidade de vida. Foram analisadas oito mulheres com faixa etária média de 66,0 0 7,3 anos que integram a Unidade Básica de saúde do bairro Brasil Novo, no município de Presidente Prudente, SP, Brasil. O programa de exercício foi realizado 3 vezes por semana, por 1 hora, com exercícios de alongamento, caminhada e fortalecimento. A qualidade de vida foi analisada pelo questionário SF-36. O domínio de Aspectos Sociais foi o que obteve um maior escore, com média de $92,19 \pm 9,30$, seguido pelos aspectos físicos com média de $84,38 \pm 29,69$ e capacidade funcional com média de $82,50 \pm 13,39$. Concluímos que mulheres inseridas em um programa de reabilitação possuem boa qualidade de vida através de procedimentos simples e de baixo custo, refletindo na importância que a UBS tem de atuar na prevenção primária e secundária.

Palavras-chave: Qualidade de vida, cardiopatias, saúde feminina.

\section{EVALUATION OF QUALITY OF LIFE IN WOMEN WITH CARDIOVASCULAR RISK FACTORS OF A BASIC HEALTH UNIT}

\begin{abstract}
The prevalence of heart disease is higher in males, however, epidemiological data show that women are achieving increasing levels. The objective of this research was to guide and act through a program of cardiac rehabilitation in women with cardiovascular risk factor inserted in a Basic Health Unit (BHU) and evaluate their quality of life. We analyzed eight women with an average age of $66.0 \pm 7.3$ years comprising the Basic Health Unit of neighborhood Brasil Novo, in the city of Presidente Prudente, SP, Brazil. The exercise program was performed 3 times a week for 1 hour with stretching exercises, walking and strengthening. Quality of life was assessed by questionnaire Short-Form 36 - Medical Outcomes Study (SF-36). The Domain Social Aspects was the one that had a higher score, with a mean of $92.19 \pm 9.30$, followed by physical with a mean of $84.38 \pm 29.69$ and functional capacity with a mean of $82.50 \pm 13.39$. It was concluded that women entered into a rehabilitation program have good quality of life through simple procedures and low cost, reflecting the importance that UBS has to work in primary and secondary prevention.
\end{abstract}

Keywords: Quality of life, cardiac, women's health 


\section{INTRODUÇÃO}

As cardiopatias atualmente ${ }^{1}$ são as maiores causadoras de óbito. Dados epidemiológicos apontam cerca de 1.150 .000 de internações por ano, devido à insuficiência cardíaca, gerando um gasto de $\mathrm{R} \$ \mathbf{4 7 5}$ milhões anualmente sem incluir gastos extras, como procedimentos de alta complexidade ${ }^{2}$. A maior prevalência está em indivíduos do sexo masculino, porém, dados epidemiológicos mostram que as mulheres estão alcançando índices cada vez maiores, graças à emancipação feminina, igualando-se aos fatores de riscos do sexo masculino: tabagismo, stress laboral, alimentação inadequada, entre outros ${ }^{3}$, sendo uma preocupante para o Ministério da Saúde. Segundo o banco de dados epidemiológicos do Ministério da Saúde, no Estado de São Paulo 11.424 indivíduos do sexo feminino tiveram óbito por alguma Doença Isquêmica do Coração, enquanto que do sexo masculino, foram 15.813 mortes no ano de 2010. Entretanto, na década de 90 esse número para o sexo feminino era de 10.602 óbitos e para o masculino 14.356 mortes ${ }^{4}$.

O Ministério da Saúde vem se mostrando preocupado com o aumento das doenças crônicas não transmissíveis, trazendo para a população programas executados pelo SUS (Sistema Único de Saúde), como o Hiperdia que consiste em um sistema de cadastramento e acompanhamento de portadores de Diabetes Mellitus e Hipertensão Arterial de pacientes de Unidades Básica de Saúde, com o intuito de adquirir, dispensar e distribuir fármacos para a população cadastrada, e consequentemente monitorizar a epidemiologia dessas doenças ${ }^{5}$.

Como a prevalência de Doenças Crônicas não transmissíveis no sexo feminino vem aumentando ${ }^{4}$ pelo crescimento dos fatores de risco acima citados, as mulheres apresentam também $70 \%$ de chances maiores de enfartarem sem fatalidade, quando comparados aos homens ${ }^{3}$.

O governo também criou programas voltados somente para população feminina. Um exemplo é o Programa de Assistência Integral a Saúde da Mulher (PAISM), que orienta mulheres, em todos os seus problemas e necessidades de saúde com assistência em todas as fases da vida como na atuação clínico ginecológica, no campo da reprodução (planejamento reprodutivo, gestação, parto e puerpério) e nos casos de doenças crônicas ou agudas ${ }^{6}$. Com um aumento significativo de doenças coronarianas nas mulheres ${ }^{4}$, a preocupação em cuidar da saúde se torna uma realidade, sendo um alerta para o Ministério da Saúde e para a população feminina. Uma alternativa para o controle destas doenças seria de 
executar programas de reabilitação cardiovascular na própria comunidade em que estas mulheres estão inseridas, através das Unidades Básicas de Saúde.

A Unidade Básica de Saúde (UBS) atende a população, sem que haja necessidade de indivíduos migrarem para hospitais e clínicas, atuando em nível de atenção primária e secundária na saúde da população ${ }^{7}$. Ela pode agir através de procedimentos simples, como atividade física, orientada e supervisionada por fisioterapeutas, que inseridos nessa UBS graças ao Núcleo de Apoio a Saúde Familiar (NASF), têm competência a realizar reabilitações cardiovasculares através de atribuições específicas, como em prescrever exercícios aeróbicos a cardiopatas ${ }^{8}$.

A aplicação de um programa de reabilitação cardiovascular na UBS proporcionaria para mulheres que apresentam fatores de risco para cardiopatias, um nível primário e secundário de atenção á saúde, visando uma melhora na qualidade de vida e capacidade funcional das mesmas, através de procedimentos simples, como exercícios aeróbicos, o que pode reduzir o número de internações e gastos públicos, proporcionando uma diminuição do número da morbidade e mortalidade por patologias coronarianas na região.
O presente estudo teve como objetivo caracterizá-las quanto aos fatores de risco cardiovasculares e avaliar a qualidade de vida das mulheres cadastradas na UBS do bairro Brasil Novo, da cidade de Presidente Prudente, SP.

\section{METODOLOGIA}

O estudo foi do tipo descritivo e de corte transversal. Foram analisadas oito pacientes do gênero feminino com faixa etária entre 55 e 73 anos que integram a UBS do bairro Brasil Novo, no município de Presidente Prudente, SP, Brasil. As mulheres participantes da reabilitação cardiorrespiratória apresentaram estabilidade clínica, com pressão arterial média (PAM) menor que $120 \mathrm{mmHg}$, sem presença de sinais e sintomas de cardiopatia aos moderados esforços.

Os critérios de inclusão foram mulheres que apresentaram estabilidade clínica, sem presença de sinais e sintomas de cardiopatia, sem limitações ortopédicas e com condições cognitivas para responderem adequadamente aos questionários.

Foram excluídas as mulheres que não apresentaram os critérios acima estabelecidos e que se recusaram em participar do estudo.

Todas as participantes assinaram o Termo de Consentimento Livre e Esclarecido, ficando cientes da pesquisa e da extração dos 
dados para o estudo. Este estudo foi realizado após a aprovação pelo Comitê de Ética em Pesquisa da Unoeste (Protocolo No. 1473)

Avaliação

Foram realizadas avaliações individuais para traçar á caracterização da amostra. Nesta ficha de avaliação obteve-se informações de cada paciente, informando os seus dados pessoais, incluindo data de nascimento, idade, estado civil, endereço, telefone escolaridade, profissão, médico responsável.

Antes e após cada sessão de treinamento cardiorrespiratório os dados hemodinâmicos foram verificados: Pressão Arterial segundo as Diretrizes de Hipertensão Arterial $^{9}$ e, consequentemente, a PAM (subtraindo a PAS sobre a PAD), FC e glicemia capilar de cada paciente.

Reabilitação

A Reabilitação Cardiovascular realizou-se um programa de exercício supervisionado por equipe interdisciplinar composta de fisioterapeuta e educador físico e constou das seguintes etapas:

Aquecimento: Antes de iniciar o exercício aeróbico, as pacientes realizaram um alongamento ativo global de toda musculatura de membros superiores e membros inferiores, com o intuito de promover uma maior flexibilidade; e uma caminhada leve do tipo trote, para aquecer e preparar o sistema muscular, cardiovascular e pulmonar para a atividade física que será iniciada ${ }^{10}$.

Condicionamento: Foi iniciado com uma caminhada leve, evoluindo para um esforço moderado. A avaliação de intensidade de exercício aeróbico foi medida através da escala de Borg onde passados os 5-10 minutos, o individuo deverá sentir-se levemente cansado, proporcionando um exercício não muito fácil, e nem cansativo ${ }^{11}$. Foram associados a essa caminhada exercícios resistidos de membros superiores composta por 2 series de 10 repetições de flexão, extensão de ombro, cotovelo e movimentos combinados em diagonais com a utilização de garrafas preenchidas com areia, composta por $1 \mathrm{Kg}$ e cordas com resistência elástica.

Desaquecimento: Após uma hora de caminhada, as pacientes realizaram uma diminuição da intensidade do exercício aeróbico. Após esse procedimento, as mesmas executaram um alongamento ativo global das cadeias musculares de membros superiores e membros inferiores com o tempo de 15 segundos associados a respiração diafragmática. 
As sessões duraram 1 hora por dia, sendo uma três vezes na semana.

Qualidade de vida

Foi avaliada através do questionário "Short-Form 36 - Medical Outcomes Study" (SF-36), que consiste em 36 itens subdivididos em oito componentes para ser avaliados: capacidade funcional, aspectos físicos, dor, estado geral de saúde, vitalidade, aspectos sociais, aspectos emocionais e saúde mental ${ }^{12}$.

Os resultados foram expressos em média ddesvio padrão, e em valores percentuais e absolutos.

\section{RESULTADOS}

Foram avaliadas 8 pacientes do gênero feminino, com média de idade de $66,0 \pm 7,3$ anos. As características clínicas e demográficas e os fatores de risco cardiovascular estão apresentados na Tabela 1.

A qualidade de vidas destas pacientes foi avaliada pelo SF-36, composto pela avaliação de 8 domínios. Cada domínio resulta em uma média de 0 a 100, onde 100 é o melhor score. Observou-se que o domínio da Vitalidade foi o mais comprometido, com uma média de $58,13 \pm 22,67$. Já o domínio de Aspectos Sociais foi o que obteve um maior escore, com uma média de 92,19 $\pm 9,30$ conforme mostra a Figura 1.

Tabela 1. Percentual das características clínicas e demográficas das mulheres do Grupo de Reabilitação Cardiovascular.

\begin{tabular}{lcc}
\hline VARIÁVEIS & (\%) & (n=8) \\
\hline Estado civil & & \\
Casadas & 87,5 & 7 \\
Viúvas & 12,5 & 1 \\
\hline Escolaridade & & \\
Ensino fund. incompleto & 100 & 8 \\
Profissão & & \\
Aposentadas & 37,5 & 3 \\
Do lar & 50,0 & 4 \\
Costureiras & 12,5 & 1 \\
\hline Patologia CVS* & & \\
HAS* & 87,5 & 7 \\
Dislipidemia & 25,0 & 2 \\
Diabetes Melittus & 25,0 & 2 \\
\hline Medicação em uso & & \\
Anti hipertensivos & 75,0 & 6 \\
Hipoglicemiantes & 12,5 & 2 \\
\hline Tabagismo & & \\
Não tabagista & 100 & 8 \\
Tabagista passivo & $37,5 \%$ & 3 \\
\hline Alcoólatra & & \\
Não & $100 \%$ & 8 \\
\hline * CVS: cardiovascular, HAS: hipertensão arterial \\
sistólica
\end{tabular}




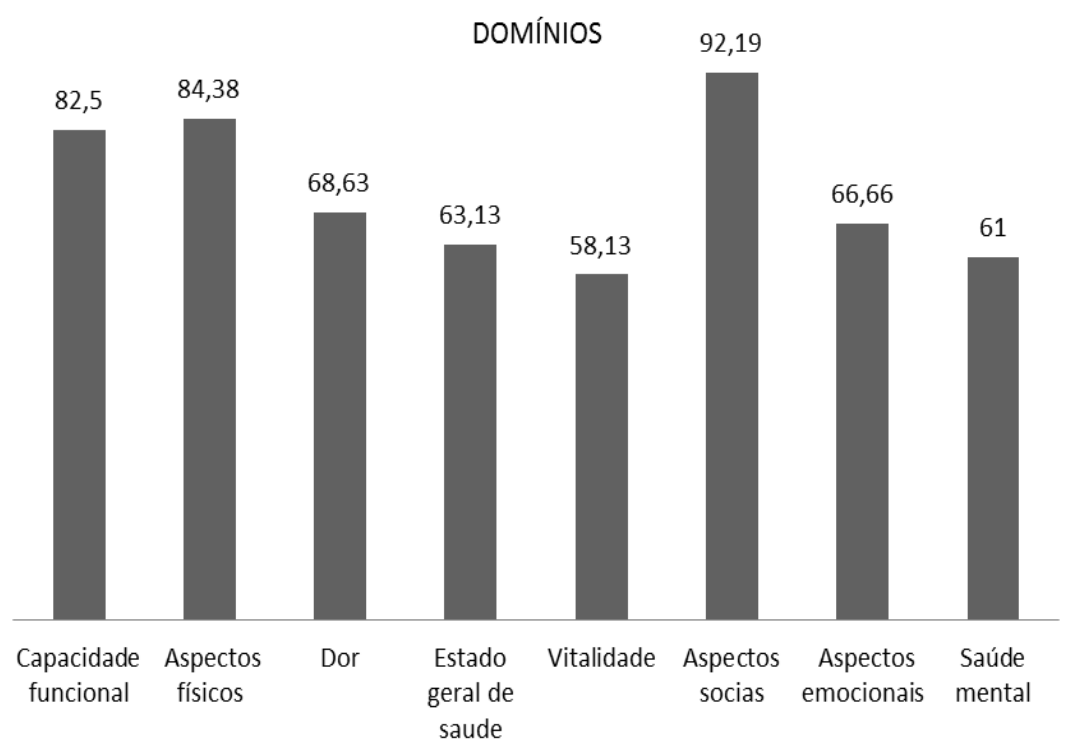

Figura 1. Valor da média de cada domínio da Qualidade de Vida (SF-36).

\section{DISCUSSÃO}

A qualidade de vida necessita de uma avaliação detalhada, por meio de diferentes domínios, pois há um conjunto de fatores como o bem estar físico, psicológico e social envolvendo- $\mathrm{a}^{13}$. O questionário escolhido pelo estudo possibilita ter uma visão global do indivíduo, pois os domínios do SF-36 proporcionam um campo de avaliação amplo por meio dos aspectos físicos (capacidade funcional, estado geral de saúde, dor, aspectos físicos) e aspectos emocionais (saúde mental, vitalidade, aspectos sociais e aspectos emocionais) ${ }^{14}$.

$\mathrm{O}$ aspecto social se destacou com maior escore neste estudo em que as atividades foram realizadas com vários indivíduos ao mesmo tempo, sendo uma estratégia para uma UBS de um bairro periférico de grande extensão. Quando se trabalha em grupo, podemos obter uma melhora da qualidade de vida, através da socialização do paciente.

O exercício físico tanto aeróbio como resistido foi realizado de forma simples em quadra coberta e foi de baixo custo, sem ergômetros como bicicletas e esteiras e sem equipamentos de musculação, e mostraramse adequados para promover uma boa qualidade de vida para estas pacientes.

A pontuação final de cada domínio do questionário SF-36 gera um escore de 0 a 100 pontos, onde zero corresponde ao pior estado de saúde do indivíduo e 100 ao seu melhor estado ${ }^{15}$. As pacientes apresentaram um escore elevado de capacidade funcional, mostrando que a reabilitação além atuar de forma preventiva a futuras complicações cardíacas, proporciona a essas mulheres que apresentam fatores de risco para essas 
doenças positivamente nas limitações funcionais e fazendo com que a prevenção primária e secundária esteja inserida dentro da realidade desta UBS. Isto implica que o exercício está contribuindo para que estas mulheres sejam independentes nas suas atividades de vida diária.

Não foi avaliada a presença de doenças osteoarticulares que são fatores preponderantes muito comuns em mulheres desta faixa etária e que ocasiona episódios álgicos ${ }^{16}$. Entretanto, estudos mostram que há correlação entre o SF-36 e o questionário OPAQ, um instrumento de avaliação específico para osteoporose na qualidade de vida de indivíduos com doenças osteoarticulares $^{17}$. Os resultados mostraram que esta reabilitação cardiovascular contribui para prevenção de limitações por aspectos físicos, por ter atingindo um alto score nesse estudo.

A amostra do estudo foi reduzida, e há necessidade de mais estudos para comprovarem a influência destes programas na qualidade de vida destas mulheres. E estudos recentes mostraram que o número de gastos públicos com doenças crônicas são altos, mas que podem ser revertidos através da atividade física ${ }^{18}$, ressaltando a importância da implantação da reabilitação em uma UBS como estratégia de diminuir gastos e ao mesmo tempo, proporcionar para a comunidade uma melhor qualidade de vida.

\section{CONCLUSÃO}

Mulheres inseridas em um programa de reabilitação possuem boa qualidade de vida através de procedimentos simples e de baixo custo, refletindo na importância que a UBS tem de atuar na prevenção primária e secundária, ampliando a multidisciplinaridade entre profissionais da área da saúde, como educador físico e fisioterapeutas somados para proporcionar bem-estar à população dessa comunidade.

\section{CONFLITO DE INTERESSES}

Os autores declaram não haver qualquer potencial conflito de interesse que possa interferir na imparcialidade deste trabalho científico.

\section{REFERÊNCIAS}

1. Mansur AP, Favarato D. Mortalidade por doenças cardiovasculares no Brasil e na região metropolitana de São Paulo. Arq Bras Cardiol. 2012;99(2):755-761. DOI: http://dx.doi.org/10.1590/s0066-

\section{X2012005000061}

2. Ministério da Saúde (Brasil), Secretaria de Ciência, Tecnologia e Insumos Estratégicos, Departamento de Ciência e Tecnologia. ELSA Brasil. Brasília: Ministério da Saúde; 2007.

3. Slopen N, Glynn RJ, Buring JE, Lewis TT, Williams DR, Albert MA. Job Strain, Job 
Insecurity, and Incident Cardiovascular Disease in the Women's Health Study: Results from a 10-Year Prospective Study. PLoS One. 2012;7(7):e40512.

4. Ministério da Saúde. DATASUS 2012. Informações sobre a saúde. [acesso em 18 ago 2012]. Disponível em: www.datasus.gov.br

5. Miranzi SSC, Ferreira FS, Iwamoto HH, Pereira GA, Miranzi MAS. Qualidade de vida de indivíduos com diabetes mellitus e hipertensão acompanhados por uma equipe de saúde da família. Texto Contexto Enf. 2008;17(4):672-679.

DOI:

http://dx.doi.org/10.1590/S0104-

\section{$\underline{07072008000400007}$}

6. Freitas GL, Vasconcelos CTM, Moura ERF, Pinheiro AKB. Discutindo a política de atenção á saúde da mulher no contexto de promoção da saúde. Rev Eletr Enf. 2009;11(2):424-428.

7. Castanheira ERL, Dalben I, Almeida MAS, Puttini RF, Patrício KP, Machado DF. Avaliação da qualidade da Atenção Básica em 37 municípios do centro-oeste paulista: característica da organização da assistência. Saúde Sociedade. 2009;18(2):84-88. DOI: http://dx.doi.org/10.1590/S0104-

\section{$\underline{12902009000600014}$}

8. Borges AMP, Salício VAMM, Gonçalves MANB, Lovato M. A contribuição do fisioterapeuta para o programa de saúde da família: uma revisão da literatura. UNIciências. 2010;14(1):69-82.

9. Sociedade Brasileira de Cardiologia / Sociedade Brasileira de Hipertensão / Sociedade Brasileira de Nefrologia. VI Diretrizes Brasileiras de Hipertensão. Arq Bras Cardiol. 2010;95(1supl.1):1-51.

10. Alencar TAMD, Matias KFS. Princípios fisiológicos do aquecimento e alongamento muscular na atividade esportiva. Rev Bras Med Esporte. 2010;16(3):230-234. DOI: http://dx.doi.org/10.1590/S1517$\underline{86922010000300015}$

11. Borg GA. Psychophysical bases of perceived exertion. Med Sci Sports Exerc. 1982;14(5):377-381.

DOI: http://dx.doi.org/10.1249/00005768198205000-00012

12. Ware Jr J, Sherbourne CD. The MOS 36Item Short-Form Health Survey (SF-36). Med Care. 1992;30(6):473-483. DOI: http://dx.doi.org/10.1097/00005650199206000-00002

13. Pimenta PAF, Simil FF, Tórres HOG, Amaral SFC, Rezende FC, Coelho OT, Rezende NA. Avaliação da qualidade de vida de aposentados com a utilização do questionário SF-36. Rev Assoc Med Bras 2008;54(1):55-56.

14. Rossi RC, Vanderlei FM, Medina LAR, Pastre CM, Padovani CB, Vanderlei LCM. Influência do perfil clínico e sociodemográfico na qualidade de vida de cardiopatas submetidos á reabilitação cardíaca. ConScientiae Saúde. 2011;10(1):5968.

DOI: http://dx.doi.org/10.5585/ConScientiaeSaud e/2011/v10n1/2465

15. Morales RR, Morales NMO, Rocha FCG, Fenelon SB, Pinto RMC, Silva CHM. Qualidade de vida em portadores de esclerose múltipla. Arq Neuropsiquiatr. 2007;65(2-B):454-460. DOI: $\quad$ http://dx.doi.org/10.1590/S0004$\underline{\text { 282X2007000300018 }}$

16. Sebastião E, Christofolleti G, Gobbi S, Hamanaka YYA. Atividade física e doenças crônicas em idosos de Rio Claro-Sp. Motriz. 2008;14(4):381-388.

17. Lemos DCM, Miyamoto TS, Valim V, Natour J. Qualidade de vida em pacientes com osteoporose: correlação entre OPAQ e 
SF-36. Rev Bras Rematol. 2006;46(5):323328. DOI: http://dx.doi.org/10.1590/S0482$\underline{50042006000500004}$

18. Bielemann MR, Knuth GA, Hallal CP. Atividade física e redução de custos por doenças crônicas ao sistema único de saúde.

Rev Bras Ativ Física Saúde. 2010;15(1):24-32.

Recebido para publicação em 16/08/2013

Aceito em 12/09/2013 